\title{
GOR'KIJ AL CINEMA: I BASSIFONDI NELLA LETTURA DI RENOIR E KUROSAWA
}

\author{
NADIA CICOGNINI
}

ABSTRACT. - Nella relazione vengono confrontati e analizzati i due adattamenti di Renoir e Kurosawa del dramma di Gor'kij I bassifondi per esaminare come all'interno di essi vengono rappresentati le idee di fondo e lo spirito del dramma gorkiano.

A tale proposito risulta interessante esaminare in entrambi i film la scelta delle scene e la rappresentazione dei personaggi e della simbologia dell'originale letterario.

Jean Renoir, che adattò il dramma di Gor'kij nel 1936 con la collaborazione di E. Zamjatin, vi trasferì molti elementi della vita contemporanea francese, volendo realizzare un dramma umano.

Akira Kurosawa trasferì l'azione del dramma dalla Russia zarista nella Tokyo dell'inizio del XIX secolo, girando quasi tutte le scene in un unico interno, nel dormitorio, e conferendo al film una sottile dimensione psicologica e uno spessore filosofico.

$$
* * *
$$

Горький и кино: "На дне" в разработке Ренуара и Куросавы

В докладе сравниваются и анализируются экранизации пьесы Максима Горького "На дне", сделанные Ренуаром и Куросавой с целью доказывать как выражают идейный замысел и дух пьесы русского писателя.

В обеих фильмах особый интерес представляют выбор сцен, трактовка персонажей и разработка символики литературного источника.

Жан Ренуар экранизировал пьесу Горького в 1936 г. с участием Е. Замятина. В нем перенес много черт современной Франции и стремился создать общечеловеческую драму.

Акира Куросава перенес действие пьесы из царской России в старый Токио начала XIX века, снимая все сцены чуть ли ни в одном интерьере, в ночлежке, наделяя фильм тонькими психологическими образами и философскими нюансами. 
Nelle sue memorie, che contengono anche un ritratto intenso e appassionato di Gor'kij, Zamjatin annotava: "Un mese e mezzo prima della sua morte, una casa di produzione di Parigi decise di girare dalla mia sceneggiatura un film ispirato alla celebre pièce di Gor'kij I Bassifondi. Gor'kij ne fu informato e giunse da lui una risposta nella quale si diceva soddisfatto della mia partecipazione al lavoro e ansioso di vedere l'adattamento del dramma e ricevere il manoscritto. Il manoscritto era già pronto, ma non poté mai essere spedito: il destinatario ci aveva ormai lasciati".

Grazie all'intervento di Gor'kij, cui lo legava una profonda amicizia, Zamjatin aveva potuto abbandonare l'Unione Sovietica nel 1931 per Parigi, dove nei difficili anni dell'esilio aveva lavorato come sceneggiatore per il cinema, collaborando con la casa di produzione Albatros dell'émigré Alexander Kamenka. ${ }^{2}$ La pièce gorkiana, accolta con successo nei più importanti teatri europei, era già nota al pubblico francese, essendo stata rappresentata nel 1905 al Théâtre de l'Oeuvre per la regia di Aurélien Lugné-Poë, con Eleonora Duse nel ruolo di Vasilisa Karpovna e nel 1922 da Georgij Pitoëff con Ljudmila Pitoëff, sua moglie, nel ruolo della prostituta Nastja e il grande Michel Simon, poi straordinario interprete de L'Atalante di Vigo, in quello del berrettaio Bubnov.

Per l'adattamento cinematografico di Bassifondi la scelta di Kamenka era caduta su Jean Renoir, che aveva già al suo attivo due trasposizioni di classici letterari, Nana, del 1926, da Zola e Madame Bovary del 1933 da Flaubert.

Erano gli anni dell'ascesa del Fronte Popolare, in cui il Partito comunista francese e le organizzazioni culturali di sinistra coagulavano intorno a sé il consenso di artisti e intellettuali, e Renoir, simpatizzante del Fronte Popolare, collaborava con "Ciné-Liberté" ed era vicino al Gruppo Ottobre, guidato da Jacques Prevert e Lou Tchimoukow, con cui aveva realizzato nel 1935 Le crime de Monsieur Lange.

1. E. Zamjatin, Ja bojus'. Literaturnaja kritika, publicistika, vospominanija, Moskva 1999, p. 232. (Zamjatin, malato di tisi, si spense di lì a poco, nel 1937).

2. La Société de films Albatros aveva prodotto, tra gli altri, nel 1923, il film Le Brasier ardent diretto dal geniale attore-regista Ivan Mosjoukine (Mozžuchin), punto di riferimento obbligato per il cinema russo dell'emigrazione (Cfr. I. Graščenkova, Kino serebrjanogo veka. Russkij kinematograf 10-ch godov i kinematograf russkogo posleoktjabr'skogo zarubežja 20-ch godov, Moskva 2005, pag. 361 e sgg). 
Les Bas-fonds, sostenuto anche finanziariamente dal Partito comunista francese, uscì sugli schermi nel dicembre del 1936, ottenendo il Premio Louis Delluc come migliore film dell'anno, e una calda accoglienza da parte del pubblico e della critica.

Tradurre in film una pièce come quella di Gor'kij, in bilico tra realismo e romanticismo, un po' dramma sociale, un po' dramma filosofico, comportava dei rischi. L'intreccio era scarno, con pochi colpi di scena piuttosto convenzionali e un epilogo tragico; tutta l'azione era focalizzata sul dormitorio, ultimo e claustrofobico rifugio di una dozzina di personaggi alla deriva: sfruttatori e ladri, assassini e alcolizzati, emarginati e diseredati, ciascuno con una sua verità esistenziale e una storia da raccontare. Un dramma corale dove tutti i personaggi sono vittime di una routine monotona e ossessiva: l'anziano usuraio e ricettatore Kostylëv, proprietario del dormitorio, la giovane e avida consorte Vasilisa, che vuole liberarsi di lui, e istiga il ladro Pepel, suo amante, a ucciderlo; la dolce e vulnerabile Nataša, sorella di Vasilisa, di cui Pepel è innamorato; la tisica Anna, moglie maltrattata del fabbro Klešč, sola nella sua triste agonia; lo scettico e lucido Satin ex galeotto; il vecchio pellegrino Luka che ha una parola di conforto per tutti; l'ex attore alcolizzato e poi suicida e la prostituta Nastja persa nei suoi sogni illusori e romantici. Tutti scherzano, si ubriacano, si azzuffano, si lamentano, parlandosi addosso, senza riuscire a comunicare davvero, e sognando impossibili evasioni da quello spazio-prigione.

È l'inferno dei bassifondi della Russia zarista e prerivoluzionaria che Gor'kij conosceva bene per esperienza diretta: per l'allestimento di Stanislavskij al Teatro d'arte di Mosca aveva predisposto un'immensa mole di annotazioni sulla vita dei criminali, dei vagabondi e dei disadattati da lui incontrati durante le sue peregrinazioni attraverso la Russia.

Il primo rischio da affrontare nella trasposizione filmica era quello dell'ambientazione. Come Stanislavskij, anche Renoir puntava a una messinscena autentica, realistica: "Non mi sono impegnato a fare un film "russo" - scriveva - ho voluto fare un dramma umano. Per comprendere meglio i personaggi di Les bas-fonds, ed essere in grado di ricrearli nel mio film, ho vagato a lungo nei sobborghi di Parigi. Là ho trovato i prototipi dei protagonisti del mio film". ${ }^{3}$ Per non ricreare una "Montmartre di samovar, balalaike, tzigani" ed evitare i soliti cliché

3. J. Renoir, La vita è cinema. Tutti gli scritti: 1926-1971, Milano 1979, p. 255. 
pseudorussi, che avrebbero banalizzato e ridicolizzato il testo narrativo gorkiano, Renoir e i suoi collaboratori (lo sceneggiatore Charles Spaak e lo scenografo Eugène Lourié) optano per un'ambientazione più neutra, meno connotata, "franco-russa" dove l'azione viene trasferita sulle rive della Senna, nei quartieri di Saint-Denis e dell'Epinay; la scenografia è parigina, ma i nomi dei personaggi restano russi e i franchi diventano rubli.

Forse la rappresentazione di un universo così autoreferenziale, chiuso, senza spiragli, come quello del dramma gorkiano, dove per i diseredati non vi è alcuna possibilità di riscatto sociale, non era tanto conciliabile col clima di fiducia del Fronte Popolare. Con un assoluto colpo di genio, Renoir trasforma il dramma in una commedia anticonvenzionale e libertaria. La dialettica dei ruoli sociali, il contrasto tra gli ambienti, i conflitti di classe restano, ma vengono trasfigurati dal suo tocco leggero e dal suo humour sottile, provocatorio.

Renoir si allontana drasticamente dalla fabula del testo gorkiano: l'intreccio ha un finale aperto, i personaggi principali si riducono a sei, mentre figure, anche importanti, come quella di Luka, che compare nel film con barba e stivali da mužik, rimangono sullo sfondo e il film si focalizza sulla dinamica del rapporto tra il barone e Pepel, interpretati dall'eccezionale coppia Jouvet-Gabin; una dinamica quasi inesistente nel dramma di Gor'kij e per cui Renoir inventa un felice prologo iniziale.

Il ladro Pepel viene sorpreso "al lavoro" di notte dal barone mentre rincasa nella sua lussuosa residenza; ma il barone, che ha sottratto un'ingente somma di denaro al Ministero, ormai ha perduto tutto, beni, reputazione e posizione sociale e nella casa non vi è più niente da rubare. Tra i due si stabilisce subito un'intesa e l'incontro diventa un momento rivelatore: il barone fa parlare il ladro e scopre che Pepel alloggia in un dormitorio pubblico, e attraverso di lui comincia a intravedere un diverso orizzonte del reale, al di fuori della rete costrittiva dei rapporti sociali e del denaro, mentre dal canto suo Pepel, che si vanta di provenire da una famiglia di ladri professionisti, comincia a dubitare della validità della propria scelta esistenziale. La lunga sequenza del dialogo, filmata attraverso un onirico gioco di ombre e di luci, oltre che un momento chiave del film, è anche un capolavoro sul piano interpretativo, per la recitazione contenuta, sottotono di Louis Jouvet e Jean Gabin.

Il barone si risveglia cambiato. Chiede al maggiordomo Felix se nella sua vita gli è mai capitato di dormire sull'erba e il maggiordomo gli risponde scandalizzato, che lui ha una dignità da difendere. L'iro- 
nico, sottile scambio di battute, anticipa già le intenzioni future del barone che si rifugerà di lì a poco nel dormitorio dove vivono Pepel e i suoi compagni derelitti e dove abbandonerà senza rimpianti il passato, ritrovando la serenità.

A differenza di Kurosawa, che durante il suo film non allontana mai la macchina da presa dallo spazio angusto del dormitorio facendone il luogo dell'azione, Renoir sceglie di girare metà delle scene in esterni, filmando i campi, il fiume, le strade e le ciminiere della banlieu parigina.

Tutto il film è giocato sul contrasto tra interno ed esterno: le scene nella fatiscente casa di mattoni di Kostylëv, con i suoi desolati interni, la cantina-dormitorio e il cortile antistante, si alternano a quelle nello spazio aperto della campagna, con la sua natura lussureggiante, come nello splendido piano sequenza della gita domenicale nella locanda in cui Pepel raggiunge Nataša e la libera dalle grinfie del commissario, suo spasimante, dove lo sguardo pittorico di Renoir ci restituisce il dinamismo sensuale della natura estiva con i suoi suoni e le sue forme e il fluido svolgersi della vita in una visione plastica della realtà.

I personaggi vanno e vengono dal triste, buio seminterrato del dormitorio verso lo spazio aperto e libero della natura: "Quando si passa all'aria aperta, - osserva nel suo saggio De Vincenti $-^{4}$ dal seminterrato dove si sommano le infelicità degli uomini, è come se si passasse da una arrière pensée di Renoir (da tutto il peso di una materia che il naturalismo zoliano ha trattato in innumerevoli pagine, depositandola nella cultura letteraria francese a cavallo tra due secoli, e che Renoir rispolvera venendo in contatto con i russi e la politica attiva) al Renoir progressivo che guarda alla vitalità degli esseri umani, alle loro inesauribili potenzialità".

In un'altra famosa scena en plein air, il barone e Pepel, beatamente sdraiati sull'erba in riva al fiume, si abbandonano al tepore della giornata estiva e alla bellezza della natura, conversando della vita. Pepel confessa al barone di non poter più vivere come ha vissuto e di aver bisogno di ossigeno, di nuovi spazi, e gli propone di fuggire con lui, ma il barone rifiuta risolutamente. Quella scena che non stava riuscendo bene, Renoir la salvò facendo correre una lumachina sul dorso della mano di Jouvet, che tiene in bocca spensierato un filo d'erba; un espe-

4. G. De Vincenti, Jean Renoir. La vita, I film, Venezia 1996, p. 153. 
diente che gli aveva consentito di approfondire la caratterizzazione del personaggio, conquistando insieme la simpatia del pubblico.

In queste sequenze Renoir sembra dare forma visiva alla poetica della vol'nica, dell'amore per la libertà e gli spazi sconfinati del primo Gor'kij, il Gor'kij dei bosjaki, dei vagabondi, che fuggono per difendere la loro dignità e la loro libertà personale, il loro sacrosanto "individualismo": personaggi ora violenti ora miti, perduti in sogni o senza speranze, che hanno lo "spazio" come "patria".

Nell'epilogo escogitato da Renoir, Kostylëv, oggetto dell'odio di tutti gli ospiti del dormitorio, viene accidentalmente ucciso nel corso di una zuffa da Pepel, accorso in difesa di Nataša. Pepel, dopo aver rotto con l'ex amante Vasilisa, fugge con Nataša, che l'aspetta all'uscita di prigione, "verso la vita". " "Allez en route!", dice Gabin/Pepel alla sua compagna, dimenticando i bassifondi e ignorando la tragedia che si è da poco consumata: il suicidio dell'attore alcolizzato.

Un finale che è un omaggio a Chaplin, che Renoir ammirava per l'inesauribile energia del suo cinema. Nell'ultima inquadratura, come in Tempi moderni (uscito in Francia nello stesso anno di Les Bas-fonds), Jean Gabin e Junie Astor, come Charlie Chaplin e Paulette Goddard, si allontanano, dopo la colazione sull'erba, in una poetica dissolvenza, lungo la strada.

Kurosawa, che conosceva il cinema di Renoir e aveva visto Les Basfonds, gira il suo Donzoko (I bassifondi) nel 1957, trasferendo l'azione in Giappone all'inizio dell'Ottocento, "verso la fine dell'era Tokugawa, quando la vita sociale e culturale ristagnava e per sfuggire alla miseria e alla scarsa libertà la gente cercava di inventarsi piccole evasioni”, ma per il resto non dimenticandosi mai dell'originale e facendone una lettura di grande rigore filologico.

Iniziato fin da ragazzo dal coltissimo fratello Heigo, morto suicida in giovane età, "la sua sola guida", alla pittura, al cinema e alla lettura, Kurosawa aveva una profonda conoscenza della letteratura russa, e soprattutto dell'opera di Dostoevskij; lo scrittore che come nessun altro aveva saputo descrivere gli abissi del cuore umano e di cui nel 1951

5. E. Bazzarelli, Massimo Gor'kij: certezze e ambiguità, in "Rassegna Sovietica", 38, 5, Roma 1987, p. 179.

6. Si intitola così l'edizione italiana de Les Bas-Fonds, tagliata e modificata dalla censura fascista. 
aveva adattato il romanzo L'Idiota, realizzando un film straordinario per complessità narrativa e intensità emozionale, che in Giappone aveva folgorato il pubblico.

L'umanista Kurosawa, che conosceva bene i bassifondi di Tokyo, raccontati in altri film precedenti, dall'Angelo ubriaco (1948), a Cane randagio (1949), a Vivere (1952), aveva già affrontato in una chiave sociale ed esistenziale, con esiti elevati sul piano estetico-narrativo, il tema degli umili, dei vinti, e della povertà, che imprigiona e rende folli e disperati gli individui respinti dalla realtà, ma in Donzoko, sceglie una dimensione filosofica, scavando nella psicologia dei singoli personaggi e meditando sul senso dell'esistenza umana. In questo film polifonico, superbamente recitato da tutti gli attori, ogni personaggio possiede un'anima, scandagliata fin nel profondo dalla "macchina da presa", spesso immobile, in lunghi piani sequenza. Nel film, come nel dramma, l'azione è ridotta all'essenziale.

Quasi seguendo le indicazioni di regia dello stesso Gor'kij, il film si apre con la scena desolante del dormitorio, inquadrato dal basso: "È l'alba, la cinepresa esplora gli spalti di un alto muraglione che cinge una profonda voragine... Seguendo la caduta dei rifiuti rovesciati da due spazzini affacciati sul parapetto la cinepresa scende a inquadrare sul fondo una miserabile baracca di legno puntellata da ogni lato: l'asilo di Rokubei/Kostylëv; per i quattro atti del dramma non usciremo da quel "fondo" d'imbuto dove una dozzina di "ex uomini" (così li definisce Gorki) hanno cercato un rifugio, una libertà, che si riveleranno alquanto illusori”.?

Kurosawa segue il dramma gorkiano scena dopo scena, dialogo dopo dialogo, ma nella sua messinscena tragica non mancano momenti di ironia e comicità surreale, come il balletto delirante, ispirato al Teatro Nô, di alcuni ospiti del dormitorio ubriachi. Il suo film è soprattutto un grande affresco interiore e una meditazione sulla condizione umana, espressa attraverso il punto di vista del personaggio di Kahei/Luka.

I temi di fondo della pièce gorkiana sono filtrati attraverso la poetica di Kurosawa. Il rapporto tra verità e menzogna, illusione e disillusione percorre tutto il film e ricorre come Leitmotiv nei dialoghi tra Yoshisaburo/Satin, ex assassino e giocatore, che ha una visione lucida e disincantata della vita e invita i compagni che non si danno pace a "rassegnarsi" e

7. A. Tassone, Akira Kurosawa, Milano 1995, p. 83. 
il pellegrino Kahei/Luka che, al contrario, invita tutti ad amare e a sognare perché i sogni, le illusioni non costano nulla e aiutano a vivere.

Lo splendido Kahei, il sorridente e saggio pellegrino, interpretato nel film da un attore comico molto amato dal pubblico giapponese, Bokuzen Hidar, che per la leggerezza e l'ironico distacco ricorda un monaco zen, incarna anche il tema della compassione. Kahei ha il dono di soffrire con gli altri e di saperli confortare con una parola incoraggiante, anche quando è una pietosa bugia. Assiste con dolcezza la sfortunata Asa/Anna, moglie del calderaio, nella sua agonia, promettendole la serenità dopo la morte, e a lei, che, come nel testo gorkiano, si stupisce di tanta tenerezza, Kahei imperturbabile risponde, con una bella metafora, che lui è "come un sassolino di fiume sballottato per tanto tempo e reso liscio e morbido dalla corrente". ${ }^{8}$

Esorta poi l'inquieto Pepel, interpretato da Toshiro Mifune, ad essere più coraggioso e a rifarsi una vita fuggendo con Nataša.

$\mathrm{E}$ in un infinito, emozionante piano sequenza della durata di 12 minuti difende dallo scherno degli altri la bella prostituta Osen/Nastja, che racconta la storia dei suoi amori e della sua vita mescolando realtà e illusione.

Kahei se ne va all'improvviso così com'era venuto, dopo che l'attore alcolizzato, che aveva incoraggiato a curarsi e a riprendere a recitare, s'impicca perché ha compreso di non potercela più fare, mentre le foglie spazzate dal vento vorticano nel cortiletto del dormitorio-rifugio; intensa metafora visiva e unico momento, in tutto il film, in cui avvertiamo la presenza della natura.

Renoir, vedendo il film di Kurosawa nel 1977, ne restò fortemente colpito e commentò "questo film è molto più importante del mio" .

\section{BIBLIOGRAFIA}

Bazin A., Che cosa è il cinema, Milano 1986.

Bazin A., Jean Renoir, Paris 1971.

8. Cfr. M. Gor'kij, Na dne, p. 130, in Polnoe sobranie socinenij: chudožestvennye proizvedenija v 25 tomach, Moskva 1968-1976, t. 7: P'esy, dramatičeskie nabroski: 18971906, Moskva, 1970): “Me ne hanno date tante perciò sono molle...”. È questa la battuta di Luka nel testo gorkiano. 
Cremonini G., Le logiche del racconto. Introduzione all'analisi narratologica dei film, Bologna-Torino 1991.

De Vincenti G., Jean Renoir. La vita, I film, Venezia 1996.

Gor'kij M., Na dne, in Polnoe sobranie sočinenij: chudožestvennye proizvedenija v 25 tomach, Moskva, 1968-1976, t. 7: P'esy, dramatič eskie nabroski: 1897-1906, Moskva 1970.

Graščenkova I. Kino Serebrjanogo veka. Russkij kinematograf 10-ch godov i kinematograf russkogo posleoktjabr'skogo zarubežja 20-ch godov, Moskva 2005.

Kraiski G. (a cura di), I formalisti russi nel cinema, Milano 1971.

Kurosawa A., L'ultimo samurai. Quasi un'autobiografia, Milano 2000.

Marsh C., Maxim Gorky: Russian dramatist, Oxford 2006.

Renoir J., La vita è cinema. Tutti gli scritti: 1926-1971, Milano 1979.

Renoir J., Ma vie et mes films, Paris 1992.

Sesonske A., Jean Renoir. The French films: 1924-1939, Cambridge, Mass. 1980.

Tassone A., Akira Kurosawa, Milano 1995.

Zamjatin E., Ja bojus': literaturnaja kritika, publicistika, vospominanija, Moskva 1999.

\section{FiLMOGRAFIA}

Les bas-fonds (1936). PRODUZIONE: Albatros Films.

Regia: Jean Renoir; Sceneggiatura: Eugène Zamiatine, Jacques Companeez; Adattamento: Jean Renoir, Charles Spaak; Fotografia: Jean Bachelet, Fedote Bourgassoff; SCENOGRAFIA: Eugène Lourie, Hugues Laurent; MusicA: Jean Wiener, Roger Desormieres; Montaggio: Marguerite Houlle; InTERPRETI PRINCIPALI: Jean Gabin (Pepel), Louis Jouvet (Il barone), Vladimir Sokoloff (Kostilev), Suzy Prim (Vasilisa), Junie Astor (Natacha), Jany Holt (Nastia), Léon Larive (Felix), Henri Saint-Isles (Kletsch), Natalie Alexeeff (Anna), Paul Temps (Satine), Robert Le Vigan (L'attore), Fernard Bercher (Il commissario), Reé Génin (Louka). Durata: 89 min. 
Donzoko (1957). Produzione: Toho

Regia: Akira Kurosawa; Sceneggiatura: Hideo Oguni, Akira Kurosawa; FoTOGRAFIA: Kazuo Yamasaki; SCEnOGRAFIA: Yoshiro Muraki; MusicA: Masaru Sato; Montaggio; Akira Kurosawa; InTERPRETI PRINCIPALI: Toshiro Mifune (Sutekichi/Pepel), Isuzu Yamada (Osugi/Vasilisa), Ganjiro Nakamura (Rokubei/Kostylëv), Kyoko Kagawa (Okayo/Nataša), Bokuzen Hidari (Kahei/Luka), Kamatari Fujiwara (l'attore), Eijiro Tono (Tomekichi/Klešč), Eiko Miyoshi (Asa/Anna), Akemi Negishi (Osen/Nastja), Koji Mitsui (Yoshisaburo/Satin). DuRATA: $137 \mathrm{~min}$. 